\title{
Physical Channel Modeling, Adaptive Prediction and Transmitter Diversity for Flat Fading Mobile Channel ${ }^{1}$
}

\author{
Shengquan $\mathrm{Hu}^{+}$, Hans Hallen ${ }^{*}$, Alexandra Duel-Hallen ${ }^{+}$ \\ ${ }^{+}$North Carolina State University \\ Dept. of Electrical and Computer Engineering \\ Center for Advanced Computing and Communication \\ Box 7914, Raleigh, NC 27695-7914 \\ "North Carolina State University \\ Physics Department \\ Box 8202, Raleigh, NC 27695-8202 \\ E-mail: Hans_Hallen@ncsu.edu
}

E-mail: shu@eos.ncsu.edu, sasha@eos.ncsu.edu

\begin{abstract}
Deterministic modeling of flat fading wireless channels using the method of images is presented. The models identify typical and worst-case channel parameter variation rates and yield datasets of channel strength vs. position, which are used to test a long-range adaptive prediction algorithm previously presented by the authors. Our physical insights and unique long-range prediction capability for the fast fading can be used in conjunction with space diversity -- including novel transmitter diversity schemes for a single receiving antenna -- or adaptive modulation to significantly reduce or eliminate the effects of deep fades in wireless communications.
\end{abstract}

\section{Introduction}

Signal fading due to the presence of several Doppler frequency shifted components is a dominant source of impairment in wireless communication. The greatest biterror rate (BER) loss and the associated high-power requirements result from "deep fades," due to destructive interference between the component radio waves [1, 2]. Since the channel changes rapidly, the transmitter and receiver are not generally optimized for current channel conditions, and thus fail to exploit the full potential of the wireless channel. Recently, several adaptive modulation methods were proposed (see, e.g. [8]). In these methods, the transmitted signal varies according to the instantaneous fading channel power. As a result, much higher bit rates relative to the conventional signaling can be achieved. These adaptive modulation methods depend on accurate channel state information, but the rapid variation of the fading channel makes feed back of the current channel estimate insufficient. To design the transmitted signal properly, future knowledge of the channel is required. For fast fading conditions, conventional fading estimation techniques cannot predict the channel sufficiently far ahead to aid adaptive modulation and coding.

In [4] and earlier papers we presented a long-term prediction algorithm that forecasts fast fading channel coefficients far ahead. When this method is combined with adaptive modulation, the performance is much better than for conventional channel estimation algorithms, especially for high vehicle speeds [5]. In this paper, we concentrate on novel physical channel models required for validating the long-term prediction algorithm. We show that a more realistic view of the fading signal results from modeling it as a deterministic process formed by the addition of several scattered components, rather than a stationary random process $[1,2,6]$. The superposition of these deterministic sinusoidal components changes rapidly as the vehicle moves, producing the familiar fast-fading signal envelope observed in practice, but the amplitude, frequency and phase of each component change on a much slower time scale. The variation of these parameters is not captured by the standard Jakes model or a stationary random process description. However, the accuracy of long-term prediction is determined by the rate of change of these parameters. Thus, to test the long-term prediction algorithm, novel channel modeling based on the method of images is required. (Note that conventional stationary models are usually sufficient for channel estimation and short-term prediction (see, e.g. [7])).

The description of our deterministic modeling method and expected channel parameter variation is contained in Section 2. To adjust to the parameter variation, the originally proposed channel prediction algorithm is augmented with adaptive parameter update as described in Section 3 (see also [5]). Finally, a transmitter diversity approach based on the proposed prediction method is presented in Section 4.

\section{Deterministic nature of the fast fading channels}

The fading signal is often modeled as a random process $[1,2,6]$ arising from an infinite number of scatterers. However, propagation studies in a variety of environments show that the fading signal consists primarily of a small number of discrete sinusoidal components (often 10 or fewer) and the interference pattern is formed from the superposition of these components $[3,6]$. Generally, we can observe this process from two different frames of reference.

(a) The first frame of reference is the ground. In this case, we assume that all reflectors are stationary and the receiver moves, so there are no Doppler shifts in this frame. The receiver passes through this interference pattern. Models of the interference pattern give insights into relative importance of scatterers, and the parameter variation rates of the component signals. The positions of destructive interference are the deep fades.

\footnotetext{
${ }^{1}$ This research was supported by CACC (NC State University) and NSF grants CCR-9725271 and NCR-9726033
} 


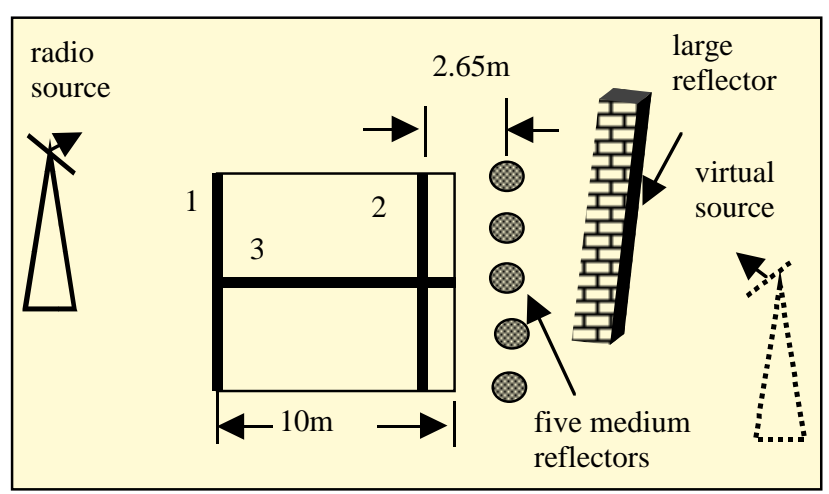

Figure 1: Generation of interference pattern of realistic physical model

(b) A different physical picture of this scenario derives from the frame of reference of the mobile rather than the ground. In this frame, the component signals are Doppler shifted, and interfere with each other in time rather than position. At the mobile, the lowpass received flat fading signal is given by

$$
c(t)=\sum_{n=1}^{N} A_{n} e^{j\left(2 \pi f_{n} t+\phi_{n}\right)}
$$

where (for the $n^{\text {th }}$ scatterer) $A_{n}$ is the amplitude, $f_{n}$ is the Doppler frequency, and $\phi_{\mathrm{n}}$ is the phase. The Doppler frequency is determined by the mobile's speed and the angle between the radio wave and the mobile's direction.

In contrast to the fast variation of fading signal $\mathrm{c}(\mathrm{t})$, the parameters $A_{n}, f_{n}$ and $\phi_{n}$ vary on much slower time scale, e.g. on the order of 100 times the coherence time of the signal envelope. This assumption can be justified from the physics based on the method of images combined with diffraction theory as discussed below and in [3]. In typical environments such as a rural highway, suburban and urban areas, it appears that most situations (but not all - base station siting is still important) would be adequately handled by a system which could respond to changes in Doppler frequency and amplitude on the 0.1 second time scale (assuming $1 \mathrm{GHz}$ carrier frequency). The rate of component phase change is slow, and is not expected to be important at all in most scattering objects realizations.

A signal is important in the interference pattern only when its strength is of comparable to the strongest component present. Accordingly, small scatters (e.g. cans, people, road signs) will not be important in the presence of the direct signal or reflections from large or medium-sized scatterers (e.g. buildings, hillsides or cars) with large amplitudes over a broad area. The treatment of reflections from large and medium-sized objects as virtual sources positioned according to curvature and diffracted through a 'slit' the size of the object in the object plane gives immediate qualitative insights into typical and worst-case rates of parameter variation. These physical insights into the nature of the fast fading provide limits on the speed of adaptation needed for an algorithm which can predict the channel significantly into the future, i.e., to reveal the timing of future deep fades and future periods with better than average channel conditions.

\section{Adaptive prediction of the flat fading channel}

A novel low rate linear prediction (LP) method for the flat fading channel was proposed in $[4,5]$ (see also earlier references in [4]). It is different from the conventional channel estimation in two aspects. First, the novel LP method focuses on predicting the future behavior of the fading coefficients rather than estimating its current value. Second, in contrast to conventional channel estimation employed at high sampling rate (usually at the data rate, e.g. [7]), the novel method used considerably lower sampling rate (on the order of twice the maximum Doppler frequency), which allows the long range channel behavior to be captured. Low rate samples are then interpolated to predict at the data rate. The LP method is based on All-Pole modeling. In this model, the predicted future channel sample $\hat{c}_{n}$ is based on $p$ previous channel samples $\mathrm{c}_{\mathrm{n}-1}, \cdots, \mathrm{c}_{\mathrm{n}-\mathrm{p}}$ :

$$
\hat{c}_{n}=\sum_{j=1}^{p} d_{j} c_{n-j}
$$

The variation in channel parameters (see Section 2) and limited record size require adjustment of the LP coefficients $\mathrm{d}_{\mathrm{j}}$ in (2). This adjustment is performed as new channel samples are received. The least mean squares (LMS) adaptive tracking method was used to update the model parameters as follows:

$$
\underline{\mathrm{d}}(\mathrm{n}+1)=\underline{\mathrm{d}(\mathrm{n})}+\eta \mathrm{e}_{\mathrm{n}} \underline{\tilde{\mathrm{c}}}_{\mathrm{n}}^{*}
$$

where $\eta$ is the step-size, $\underline{d}(n)=\left(d_{1}(n), \ldots d_{p}(n)\right)$ is the timedependent vector of channel model parameters (see (2)),

$\tilde{\tilde{c}}(\mathrm{n})=\left(\tilde{\mathrm{c}}_{\mathrm{n}-1}, \ldots, \tilde{\mathrm{c}}_{\mathrm{n}-\mathrm{p}}\right)$ is the vector of updated channel estimates, and the error signal, $\mathrm{e}_{\mathrm{n}}=\mathrm{c}_{\mathrm{n}}-\hat{\mathrm{c}}_{\mathrm{n}} \approx \tilde{\mathrm{c}}_{\mathrm{n}}-\hat{\mathrm{c}}_{\mathrm{n}}$. In this paper we set

$\tilde{\mathrm{c}}_{\mathrm{n}}=\mathrm{c}_{\mathrm{n}}$ in the computation of $\mathrm{e}_{\mathrm{n}}$ and assume that noise-free observations are available. In [5], we show how an estimate $\tilde{\mathrm{c}}_{\mathrm{n}}$ can be computed in the presence of noise using decisiondirected LMS algorithm in conjunction with truncated channel inversion power control technique [8]. The improved parameters $\underline{d}(n)$ can be used in the modified iterative autoregressive equation to predict future channel samples, e.g.:

$$
\hat{c}_{n+r}=\sum_{j=1}^{r-1} d_{j}(n+1) \hat{c}_{n-j+r}+\sum_{j=r}^{p} d_{j}(n+1) \tilde{c}_{n-j+r}, r=1 \ldots R
$$

where $\mathrm{R}$ is the prediction range, i.e., it is desired to predict up to $\mathrm{R}$ samples ahead. In this paper, we set $\mathrm{R}=1$. Depending on the sampling and data rates, this implies prediction for tens to hundreds of data bits ahead. Application of this adaptive tracking method significantly improves accuracy and maintains the robustness of long range prediction as the physical channel parameters vary.

Although in (2) the complex fading coefficient is predicted, a similar algorithm can be developed for predicting the power of the fading coefficient $\left|c_{n}\right|^{2}$. In practice, the power (or magnitude) is the most important parameter to predict, since it directly reveals the locations of deep fades and signal peaks. Numerical results of this paper are performed assuming prediction of power only. 


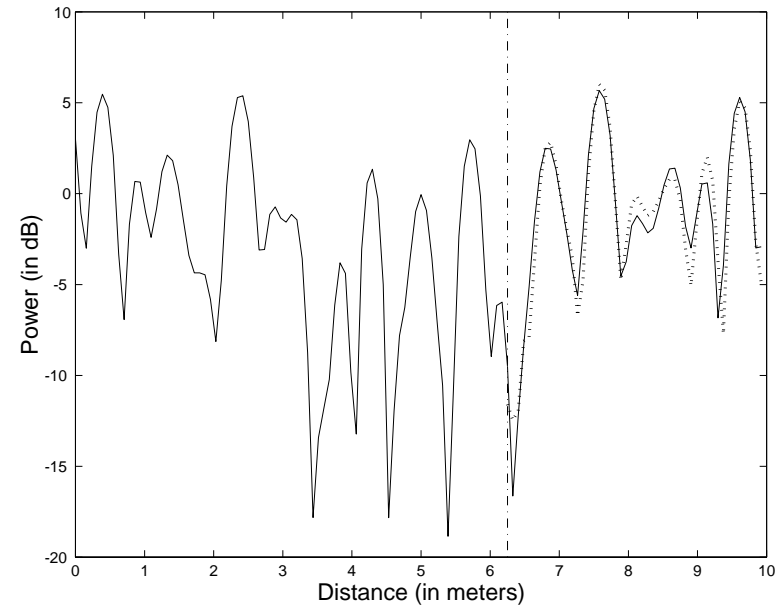

Figure 2 Parallel to and far from the reflectors (route 1). First half: the actual fading channel envelope (solid line) is observed. Second half: the actual future (solid) and predicted (dotted) fading channel envelopes. 1.28 samples/m.

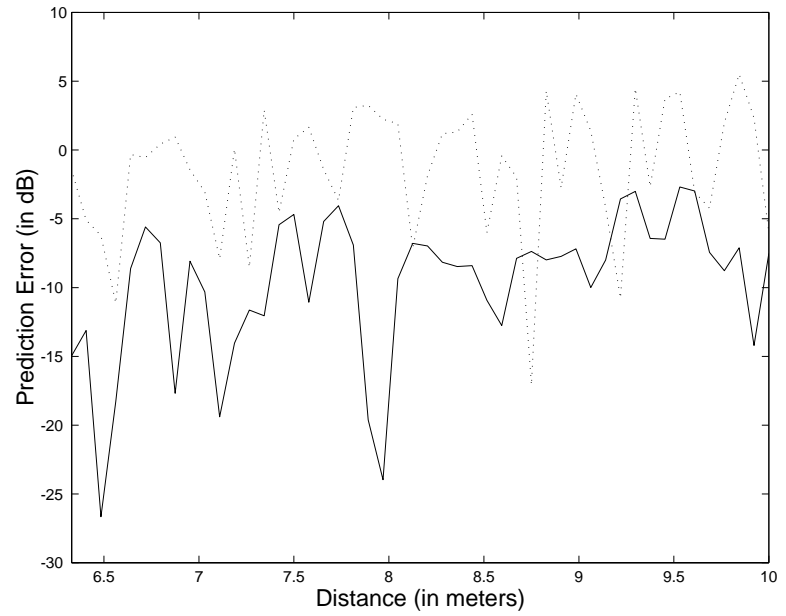

Figure 4 Prediction error comparison. Solid: route 1; Dotted: route 3. $1.28 \mathrm{samples} / \mathrm{m}$.

\section{Testing with realistic physical channel models}

As an example of deterministic modeling, suppose one large and five medium-sized reflecting objects create an interference pattern with the source. The source is $100 \mathrm{~m}$ to the left of the square region shown in Figure 1. A large hill or building $10 \mathrm{~m}$ to the right of the square region is tilted from vertical, placing its effective source $130 \mathrm{~m}$ to the right of the bottom edge of the square. Its amplitude reflection coefficient is $2 / 3$. The five medium reflectors are evenly spaced on a $10 \mathrm{~m}$ vertical line with effective sources $1 \mathrm{~m}$ to the right. Think of them as five spherical cars parked along the road, with their curvature locating the effective sources [3]. The interference pattern (e.g., Figures 2, 3) is complex with narrow, deep fades which are $\sim 1 / 100$ the average power, as is typically observed with flat fading. Variations of Doppler shifts and amplitudes with position for each of the scattering components are known, and can be used to estimate required adaptive tracking performance. In general, the rate of Doppler frequency variation (e.g. $190 \mathrm{~Hz} / \mathrm{second}$

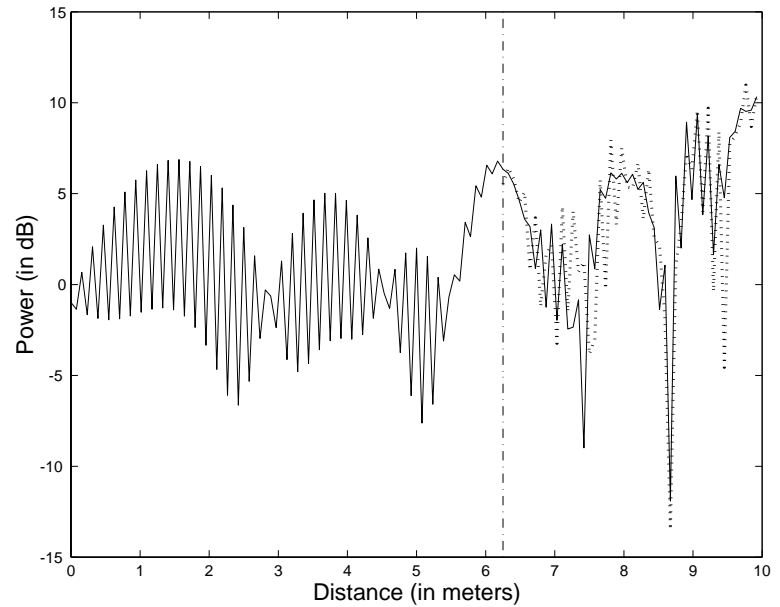

Figure 3 Perpendicular to the reflectors (route 3). First half: the actual fading channel envelope (solid line) is observed. Second half: the actual future (solid) and predicted (dotted) fading channel envelopes. 1.28 samples/m

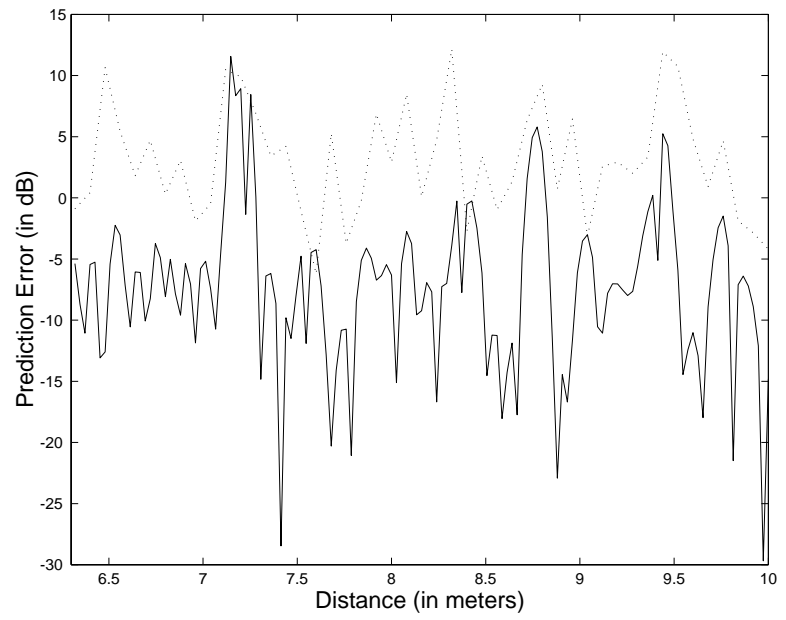

Figure 5 Prediction error comparison for route 2 with different sampling rates. Solid: 3.75 samples/m; dotted: 1.25 samples/m.

for route 1 and $406 \mathrm{~Hz} / \mathrm{second}$ for route 2 [3]) will be larger if one passes closer to the virtual source, so routes 2 and 3 represent near-worst-case scenarios. The amplitude variation is also strong along these paths, due to its inverse distance dependence.

The proposed linear prediction method was tested on the realistic physical channel models. The datasets were generated based on the configuration shown in Figure 1. We assumed that the carrier frequency was $1 \mathrm{GHz}$. If the vehicle speed was $60 \mathrm{miles} / \mathrm{h}$, the maximum Doppler frequency shift was $90 \mathrm{~Hz}$. We generated 128 samples in each 10 meter path (routes 1-3 in Fig. 1), corresponding to the channel sampling rate of $342 \mathrm{~Hz}$. We chose route 1 as a typical case and route 3 as the worst case in Fig. 1 to be tested. The observation interval was 80 samples (6.25 meters), and model order $\mathrm{p}=30$. The simulation results are shown in Figures 2-4. We see that the channel power variation can be predicted very closely for a typical situation (route 1) shown in Fig. 2. However, the prediction becomes more difficult for the route 3 due to the 


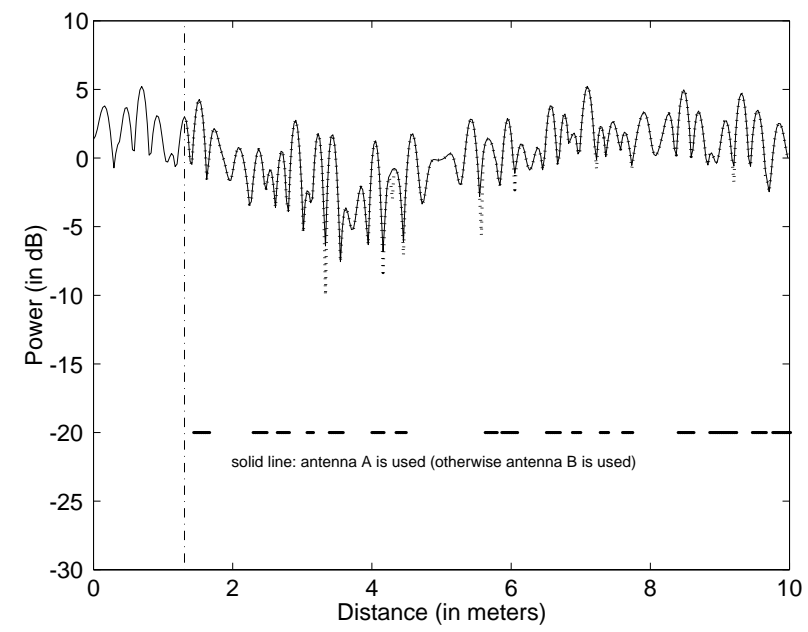

Figure 6 An example of transmitter diversity system. Part before dash line: the actual physical channel power variation (solid line) is observed. part after dash line: the maxim of the two patterns chosen by using actual values (solid line) and by using predicted values (dotted line).

large Doppler frequency shift and the large power variation as the mobile approaches the reflectors. The prediction error comparison for routes 1 and route 3 is shown in Fig. 4. In addition, in Fig. 5, we consider power prediction for another near-worst case (route 2 in Fig. 1).

In Fig. 5, prediction error is compared for two different sampling rates (the solid curve corresponds to $1000 \mathrm{~Hz}$ and the dotted to $333 \mathrm{~Hz}$ when assuming vehicle speed is 60 miles/h). Better performance results when the channel sampling rate is increased, since the parameters $\underline{\mathrm{d}}(\mathrm{n})$ in (3) can be updated more often, and better convergence results. Of course, when the sampling rate is increased significantly (e.g. comparable to the data rate), but the model order $\mathrm{p}$ is fixed, prediction range eventually decreases, since it becomes impossible to capture long-term channel behavior [5]. In general, the sampling rate, model order $\mathrm{p}$ and the choice of the adaptive algorithm have to be optimized to insure desirable accuracy, prediction range and complexity tradeoff.

\section{Transmitter diversity for a single receiving antenna}

The slow variation of fading channel parameters allows us to accurately predict future channel properties far ahead, as shown above. This prior knowledge can be used for transmitter optimization, and is especially important for the downlink since usually only one antenna is used at the mobile. For example, transmitter diversity in the downlink can be provided by adaptively selecting at the base station the transmission antenna that results in the strongest signal at the mobile. Antenna selection can be aided by the proposed prediction algorithm. For example, suppose that just two antennas A and B spaced at half wavelength apart are used at the base station. In modeling this transmitter diversity system, it is important to take into account that shifting the source antenna does not simply shift the interference pattern, since the positions of the effective sources for the reflected light will also change for flat objects. Assume that the carrier frequency is $1 \mathrm{GHz}$ and mobile drives along route 2 in Fig. 1 at the speed of $30 \mathrm{miles} / \mathrm{h}$. The fading channel is sampled at $500 \mathrm{~Hz}$, and data transmission rate is $25 \mathrm{Kbits} / \mathrm{second}$. The driving configuration is: (1) predict future channel power when transmitting from antenna $\mathrm{A}$; (2) predict future channel power when transmitting from antenna B; (3) select and use the antenna which results in the larger signal at the receiver. The resulting predicted channel power is shown in Fig. 6. As expected, this selection diversity method significantly reduces the severity of fading relative to the single antenna case (compare with Figures 2 or 3). Observe very close agreement between the actual and the predicted power. In this example, there were 33 antenna switches (refer to the line in Fig. 6) during transmission of 16250 bits. This switching rate is realistic, but could be reduced further if desired. The predicted power values can be used to decide when to switch according to a specified strategy.

Without prediction, outdated channel estimates have to be fed back from the receiver to the transmitter and used for antenna selection. However, for fast vehicle speeds this method would require very frequent feedback due to rapid channel variation. We are currently in the process of comparison of various feedback and estimation techniques, and preliminary results indicate that use of prediction provides significant performance gains. Many questions related to this approach are still open and are under investigation.

\section{Conclusions}

Realistic physical models are studied and it is observed that channel parameters vary slowly enough for tracking and long range prediction. This was examined through the channel power prediction for a realistic model. Such capability will engender transmitter optimization using simple transmitter diversity.

\section{References}

[1] T.S. Rappaport, Wireless Communications, Principles and Practice, Prentice-Hall, 1996.

[2] J.G. Proakis, Digital Communications, 3rd Ed, McGraw Hill, 1995. [3] H. Hallen, S. Hu, A. Duel-Hallen, "Physical Insights into the Nature of the Fast Fading in Wireless Communications," to be submitted to JSAC, 1999.

[4] T. Eyceoz, A. Duel-Hallen, H. Hallen, "Deterministic Channel Modeling and Long Range Prediction of Fast Fading Mobile Radio Channels," IEEE Comm. Lett., Vol.2, No.9, pp. 254-256, Sept. 1998 [5] T. Eyceoz, S. Hu, A. Duel-Hallen, H. Hallen, "Adaptive Prediction, Tracking and Power Adjustment for Frequency Non Selective fast Fading Channels," Proc. of CTMC'99, ICC'99.

[6] W.C. Jakes, Microwave Mobile Communications, Wiley, New York ,1974.

[7] Y. Liu and S. D. Blostein, "Identification of Frequency Non selective Fading Channels Using Decision Feedback and Adaptive Linear Prediction," IEEE Trans. on Commun., Vol. 43, No. 2/3/4, pp. 1494 - 1492, Feb./March/April 1995.

[8] A. J. Goldsmith and S. G. Chua, "Variable-Rate Variable-power MQAM for Fading Channels", IEEE Trans. Comm.. , Vol. 45, No. 10, pp. 1218 - 1230, Oct. 1997. 